\title{
SU(6) Grand Unification of 3-3-1 Model
}

\author{
Frank F. Deppisch ${ }^{1}$, Chandan Hati ${ }^{2,3}$ Sudhanwa Patra $^{4}$, Utpal Sarkar ${ }^{5}$, and \\ José W.F. Valle \\ 1 Department of Physics and Astronomy, University College London, \\ London WC1E 6BT, United Kingdom \\ 2 Theoretical Physics Division, Physical Research Laboratory, \\ Ahmedabad 380009, India \\ 3 Indian Institute of Technology Gandhinagar, Ahmedabad 382424, India \\ E-mail of the corresponding author : chandan@prl.res.in \\ 4 Center of Excellence in Theoretical and Mathematical Sciences, \\ Siksha 'O' Anusandhan University, Bhubaneswar-751030, India \\ 5 Department of Physics, Indian Institute of Technology Kharagpur, \\ Kharagpur 721302, India \\ 6 AHEP Group, Institut de Fisica Corpuscular - C.S.I.C./Universitat de Valencia, \\ Parc Cientific de Paterna \\ C/ Catedràtico Josè Beltràn, 2 E-46980 Paterna (Valencia) - Spain
}

\begin{abstract}
We discuss a sequential variant of the $\mathrm{SU}(3)_{\mathrm{c}} \otimes \mathrm{SU}(3)_{\mathrm{L}} \otimes \mathrm{U}(1)_{\mathrm{x}}$ model which can fit within a minimal $\mathrm{SU}(6)$ grand unification. Interestingly, this minimal $\mathrm{SU}(6)$ embedding can allow a $\mathrm{SU}(3)_{\mathrm{c}} \otimes \mathrm{SU}(3)_{\mathrm{L}} \otimes \mathrm{U}(1)_{\mathrm{x}}$ symmetry breaking scale within the reach of LHC and with seesaw-type neutrino masses.
\end{abstract}

Keywords: grand unification, 3-3-1 model, SU(6)

Introduction: The $\mathrm{SU}(3)_{\mathrm{c}} \otimes \mathrm{SU}(3)_{\mathrm{L}} \otimes \mathrm{U}(1)_{\mathrm{X}}$ [1] gauge extension of the SM provides a strong promise of new physics that can be observed at the LHC or the next generation accelerators $[2,3]$ and also can provide novel ways to understand neutrino masses . The $\mathrm{SU}(3)_{\mathrm{c}} \otimes \mathrm{SU}(3)_{\mathrm{L}} \otimes \mathrm{U}(1)_{\mathrm{X}}$ model proposed by Singer, Valle and Schechter (SVS) [2] is not anomaly free for each generation of fermions, however, when all the three generations of fermions are included the theory becomes anomaly free. Consequently, different multiplets of the $\mathrm{SU}(3)_{\mathrm{c}} \otimes \mathrm{SU}(3)_{\mathrm{L}} \otimes \mathrm{U}(1)_{\mathrm{X}}$ group appear with different multiplicity and it becomes difficult to unify the model within usual grand unified theories. In Ref. [4] we have studied how such a theory can be unified in a $\mathrm{SU}(6)$ gauge theory that can emerge from a $\mathrm{E}(6)$ Grand Unified Theory (GUT). Interestingly, the SVS 3-3-1 model can readily be refurbished into an anomaly free multiplet structure which can be right away embedded in a minimal anomaly free combination of representations of $\mathrm{SU}(6)$ as an E(6) subgroup. We refer to this new 3-3-1 model as the sequential 3-3-1 model. This scheme is particularly intriguing since this $\mathrm{SU}(6)$ embedding does not require any bulk exotics to account for the chiral families; and in that sense it provides a truly minimal unification scenario in the same spirit akin to the minimal SU(5) construction [5]. 
The sequential $\mathrm{SU}(3)_{\mathrm{c}} \otimes \mathrm{SU}(3)_{\mathrm{L}} \otimes \mathrm{U}(1)_{\mathrm{X}}$ Model: In this model we assign the fields in such a way that the anomalies are cancelled for each generation separately. The multiplet structure is given by

$$
\begin{aligned}
Q_{a L} & =\left(u_{a L}, d_{a L}, D_{a L}\right)^{T} \equiv[3,3,0], u_{a R} \equiv[3,1,2 / 3], d_{a R} \equiv[3,1,-1 / 3], \\
D_{a R} & \equiv[3,1,-1 / 3], \psi_{a L}=\left(e_{a L}^{-} \nu_{a L} N_{a L}^{1}\right)^{T} \equiv\left[1,3^{*},-1 / 3\right], \\
\xi_{a L} & =\left(E_{a L}^{-}, N_{a L}^{2}, N_{a L}^{3}\right)^{T} \equiv\left[1,3^{*},-1 / 3\right], \chi_{a L}=\left(N_{a L}^{4}, E_{a L}^{+}, e_{a L}^{+}\right) \equiv\left[1,3^{*}, 2 / 3\right] .
\end{aligned}
$$

In order to drive symmetry breaking and generate the charged fermion masses, we assume a Higgs sector similar to the SVS 3-3-1 model. The Yukawa Lagrangian for the quark sector is given by

$$
\mathcal{L}_{\text {quarks }}=y_{u_{a}} \overline{Q_{a L}} u_{a R} \phi_{0}^{*}+y_{d_{a}}^{i} \overline{Q_{a L}} d_{a R} \phi_{i}^{*}+y_{D_{a}}^{i} \overline{Q_{a L}} D_{a R} \phi_{i}^{*}+\text { h.c. } \quad,
$$

where $a=1,2,3, i=1,2$ (neglecting any flavour mixing). In the leptonic sector the relevant Yukawa interactions are given by

$\mathcal{L}_{\text {leptons }}=\epsilon_{\alpha \beta \gamma}\left[\psi_{\alpha L}^{T} C^{-1}\left(y_{1} \xi_{\beta L} \phi_{0 \gamma}+y_{2}^{i} \chi_{\beta L} \phi_{i \gamma}\right)+\xi_{\alpha L}^{T} C^{-1} y_{3}^{i} \chi_{\beta L} \phi_{i \gamma}\right]+$ h.c.

where $\alpha, \beta, \gamma$ are the $\mathrm{SU}(3)_{\mathrm{L}}$ tensor indices giving antisymmetric Dirac mass terms, $C$ is the charge conjugation matrix, and $i=1,2$. After the symmetry breaking, the $5 \times 5$ neutrino mass matrix for each generation can be diagonalized to obtain two quasi-Dirac heavy neutrinos with mass around $\mathrm{SU}(3)_{\mathrm{c}} \otimes \mathrm{SU}(3)_{\mathrm{L}} \otimes \mathrm{U}(1)_{\mathrm{X}}$ symmetry breaking scale, two Dirac states with mass of the order of the electroweak symmetry breaking scale and a light seesaw Majorana neutrino.

SU(6) Grand Unification: It is easy to verify that each generation of the fermionic multiplets of the sequential 3-3-1 model written in Eq. (1) can be embedded perfectly in the anomaly free combination of $\mathrm{SU}(6)$ representations: $\overline{6}+\overline{6}^{\prime}+15$, where $\overline{6}$ contains $d_{L}^{c} \equiv[3,1,-1 / 3]$ and $\psi_{L} \equiv\left[1,3^{*},-1 / 3\right] ; \overline{6}^{\prime}$ contains $D_{L}^{c} \equiv[3,1,-1 / 3]$ and $\xi_{L} \equiv\left[1,3^{*},-1 / 3\right] ;$ and 15 contains $u_{L}^{c} \equiv\left[3^{*}, 1,-2 / 3\right]$, $\chi_{L} \equiv\left[1,3^{*}, 2 / 3\right]$ and $Q_{L} \equiv[3,3,0]$. Now the $\mathrm{E}(6)$ fundamental representation 27 decomposes under the maximal $\mathrm{SU}(2) \otimes \mathrm{SU}(6)$ subgroup as $27=[2, \overline{6}]+[1,15]$. Three $27 \mathrm{~s}$ of $\mathrm{E}(6)$ containing three sets of $\overline{6}+\overline{6}^{\prime}+15$ can accommodate three generations of the fermionic multiplets of the sequential 3-3-1 model. However, the minimal content of the sequential 3-3-1 model does not lead to a successful unification scenario. Interestingly, by adding three generations of the fermionic octets leads to a successful gauge coupling unification.

The one-loop beta-coefficients for the phase between the electroweak symmetry breaking and the $\mathrm{SU}(3)_{\mathrm{c}} \otimes \mathrm{SU}(3)_{\mathrm{L}} \otimes \mathrm{U}(1)_{\mathrm{X}}$ symmetry breaking $\left(M_{Z}\right.$ to $\left.M_{X}\right)$ are given by $b_{2 L}=-19 / 6, b_{Y}=41 / 10, b_{3 C}=-7$. The one-loop beta-coefficients for the phase between the $\mathrm{SU}(3)_{\mathrm{c}} \otimes \mathrm{SU}(3)_{\mathrm{L}} \otimes \mathrm{U}(1)_{\mathrm{X}}$ symmetry breaking scale and the octet mass scale $\left(M_{X}\right.$ to $\left.M_{8}\right)$ are given by $b_{3 L}=-9 / 2, b_{X}=13 / 2$, $b_{3 C}^{331}=-5$. Finally, he one-loop beta-coefficients for the phase between the octet mass scale to the unification scale $\left(M_{8}\right.$ to $\left.M_{U}\right)$ are given by $b_{3 L}^{8}=2 n-9 / 2$, where $n$ is the number of generations of the fermionic octets $\left(\Omega \equiv\left[1,8^{*}, 0\right]\right)$, 


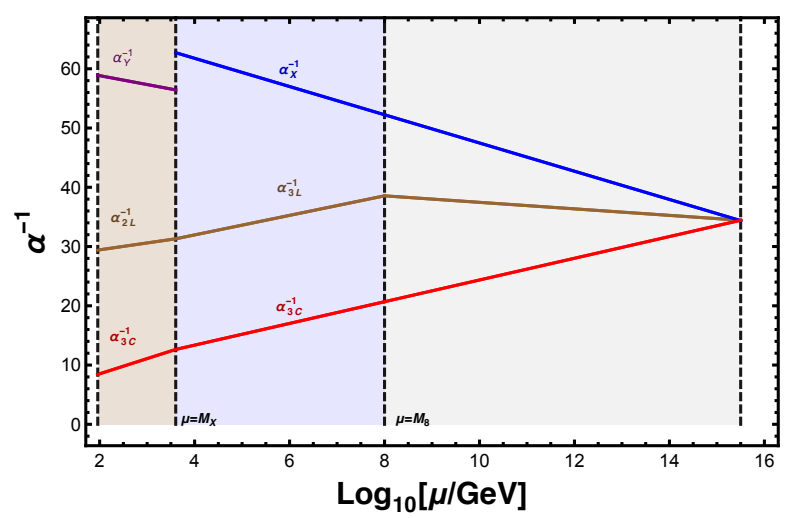

Fig. 1. The running of the gauge couplings in the sequential 3-3-1 Model with three generations of fermionic octets with $\mathrm{SU}(3)_{\mathrm{c}} \otimes \mathrm{SU}(3)_{\mathrm{L}} \otimes \mathrm{U}(1)_{\mathrm{X}}$ symmetry breaking scale $M_{X}=4000 \mathrm{GeV}$ and octet mass scale $M_{8}=10^{8} \mathrm{GeV}$, demonstrating successful gauge unification at the scale $M_{U}=10^{15.5} \mathrm{GeV}$ with $n_{Y}=\sqrt{5 / 3}$ and $n_{X}=2 / \sqrt{3}$.

$b_{X}^{8}=13 / 2, b_{3 C}^{8}=-5$. Fig. 1 shows the gauge coupling running of the sequential $\mathrm{SU}(3)_{\mathrm{c}} \otimes \mathrm{SU}(3)_{\mathrm{L}} \otimes \mathrm{U}(1)_{\mathrm{X}}$ model and three generations of fermionic octets with $\mathrm{SU}(3)_{\mathrm{c}} \otimes \mathrm{SU}(3)_{\mathrm{L}} \otimes \mathrm{U}(1)_{\mathrm{X}}$ symmetry breaking scale $M_{X}=4000 \mathrm{GeV}$ and octet mass scale $M_{8}=10^{8} \mathrm{GeV}$, demonstrating successful gauge unification at the scale $M_{U}=10^{15.5} \mathrm{GeV}$ with $n_{Y}=\sqrt{5 / 3}$ and $n_{X}=2 / \sqrt{3}$. Taking $M_{U}=10^{15.5} \mathrm{GeV}$ and $\alpha_{\mathrm{GUT}}^{-1} \sim 35$ in sequential 3-3-1 model, the lifetime of the proton decay mode $p \rightarrow e^{+} \pi^{0}$ is roughly $\sim 10^{34}$ yrs, which is consistent with the current experimental limit [6].

Concluding Remarks: We have discussed a minimal SU(6) grand unification of the sequential variant of the $\mathrm{SU}(3)_{\mathrm{C}} \otimes \mathrm{SU}(3)_{\mathrm{L}} \otimes \mathrm{U}(1)_{\mathrm{X}}$ model which allows for a $\mathrm{TeV}$ scale $\mathrm{SU}(3)_{\mathrm{c}} \otimes \mathrm{SU}(3)_{\mathrm{L}} \otimes \mathrm{U}(1)_{\mathrm{X}}$ model as well as seesaw-induced neutrino masses. The gauge coupling unification can be associated to the presence of sequential leptonic octets at some intermediate scale between the 3-3-1 scale and the unification scale. The presence of the octet scale can have interesting implications for radiative origin of neutrino masses [7].

\section{References}

1. H. Georgi, A. Pais, Phys. Rev. D19, 2746 (1979). DOI 10.1103/PhysRevD.19.2746

2. M. Singer, J. Valle, J. Schechter, Phys.Rev. D22, 738 (1980). DOI 10.1103/ PhysRevD.22.738

3. J.W.F. Valle, M. Singer, Phys. Rev. D28, 540 (1983)

4. F.F. Deppisch, C. Hati, S. Patra, U. Sarkar, J.W.F. Valle, Phys. Lett. B762, 432 (2016). DOI 10.1016/j.physletb.2016.10.002

5. H. Georgi, S. Glashow, Phys.Rev.Lett. 32, 438 (1974)

6. K. Olive, et al., Chin.Phys. C38, 090001 (2014). DOI 10.1088/1674-1137/38/9/ 090001

7. S.M. Boucenna, R.M. Fonseca, F. Gonzalez-Canales, J.W.F. Valle, Phys. Rev. D91(3), 031702 (2015). DOI 10.1103/PhysRevD.91.031702 